\title{
Research of the Technology and Application of 3D Model Based Assembly Structural Process Design for Spacecraft
}

\author{
Bi Le Wan ${ }^{1}$, Wen Xing $\mathrm{He}^{1, a}$, and Chang Yu Chen ${ }^{1}$ \\ ${ }^{1} 1$ Beijing Institute of Spacecraft Environment Engineering, Beijing 100094, China
}

\begin{abstract}
In order to implement spacecraft assembly based on MBD, this article proposes a method of structural process design based on 3D model. First, an Assembly Digital Mock-up (ADMU) is constructed. Second, we propose the way of assembly process design based on 3D structural process design scheme. Finally, the paper describes the application of the technology, and analyses the effect of the scheme during a satellite development. The technology and systems should be applied on other sophisticated products development, with some essential modifications.
\end{abstract}

\section{Introduction}

Assembly, Integrated and Test (AIT) is critical for spacecraft development. During an AIT process, Computer Aided Process Planning (CAPP) system and Kanban system are widely used for editing process files. As traditional process files consist of only texts and pictures, it is difficult for process designers to deliver product model, Bill of Material (BOM), product property and inner relationship between these information. Furthermore, based on traditional method, it is impossible to integrate and manage assembly process plan with $3 \mathrm{D}$ CAD model, and make alteration and data-package automatically. With the development of Model Based Definition (MBD), potential solutions to above problems are proposed [1-4].

Researchers described much technology, such as data definition based model [5-6], digital mock-up construction [7-8], process planning and tools design based 3D models [9-11]. And they didn't describe automatic construction of assembly data-package, which is based on 3D structural process design.

In order to fulfill the requirement of the spacecraft assembly, this article proposes a design method for 3D structural process scheme, which is based on the Assembly Digital Mock-up (ADMU) defined in [7]. Three keys of designing the scheme are presented. In order to construct assembly data-package, this article then describe integration method of Manufacture Executing System (MES) and 3D Structural Process Design System (3DSPDS). At last, effects of an application are discussed.

\section{Development of Satellite Digitization}

\subsection{Model of satellite development.}

For the characteristic of satellite production primarily comes about small scale production or single-piece production. Even if custom orders two satellites belonged to the same load platform, each satellite is manufactured in its unique way according to specific requirements. In other words, satellite is essentially produced in singlepiece way.

Usually, Satellite product organizations consist of three departments: design department, manufacturing department and AIT department. AIT department dedicates to assembly and integrated test including final assembly process design, assembly implementation, test and so on.

\subsection{Design of Three-Dimensional.}

As design department design satellite, they need to use both software Pro/E and CATIA. In the area of data management, four data management are used by design department to be employed in 3D modeling, technical file, $2 \mathrm{D}$ drawing and so on; In the area of digitization of development research and manufacturing, some satellite adopt full-specialty 3D development research and manufacturing, a few others adopt semi-specialty 3D development research and manufacturing; In the area of digitization standard system of development research and manufacturing, design standards following by different design departments are not identical.

In addition, 2D design data include technical textformatted files, word-formatted files, 2D CAD drawing and so on. There are other design data, for instance design update files, technical notes and troubleshooting file.

\subsection{Digitization of satellite assembly.}

In development research and manufacturing of spacecraft AIT, assembly department had already built digitization mock-up and created specialized process for spacecraft assembly. On the one hand it inherit Engineering Bill of Material (EBOM). On the other hand adjustment and complement would be added on EBOM. Process assembly, tools and process protection parts are

\footnotetext{
${ }^{\mathrm{a}}$ Corresponding author: hewenxing@163.com
} 


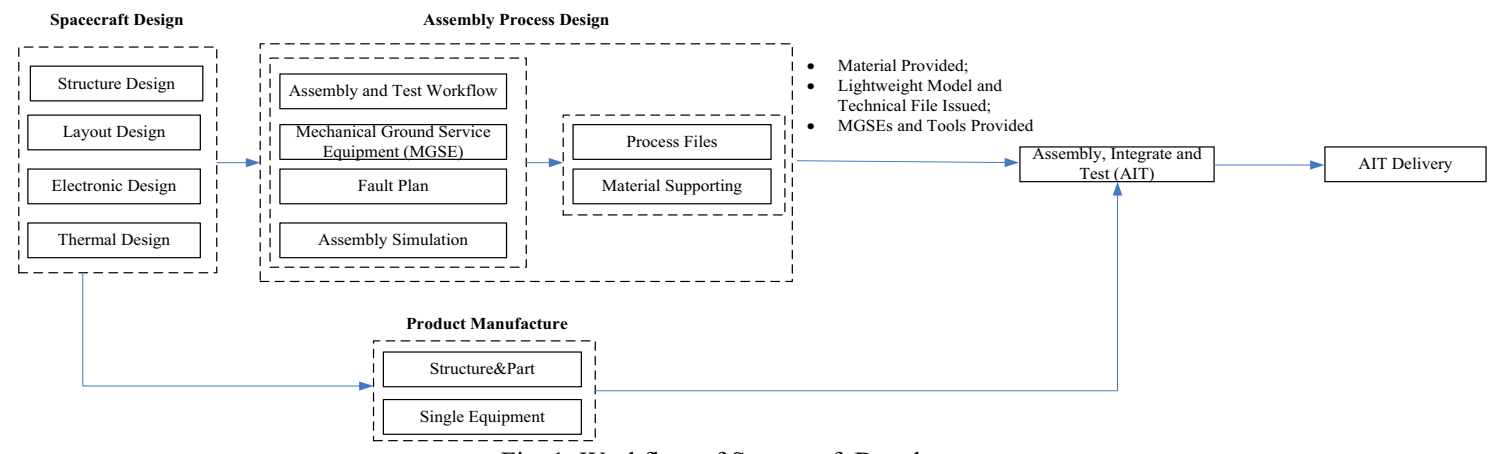

Fig. 1. Workflow of Spacecraft Development

added on base of EBOM. Same as EBOM, process digital mock-up include three components: Bill of Material, Model and Products attributes. Bill of Materiel use for delivery material information. Assembling relationship between parts and main object use for assembly technical conditions control. Model including layout, label and PMI is obtained after design lightweight. It mainly offer a good guidance field production and data sources of process design. For instance, assembly operation simulation and added process requirement. Process design depends on products attributes that had recorded assembly technology requirements, administrative information and so on.

\section{Analysis of 3D Structural Process}

Building bidirectional relationship between process design and production implementation is the prime condition of successful assembly for spacecraft using 3D
Building bidirectional relationship between process design and production implementation depends on communication with information which include design requirements, design model, simulation videos, picture and so on. Using development research and manufacturing mode, 3D process files are regarded as carrier for communication. In other words, compared with traditional two-dimensional process files, 3D process files not only disseminate information by text, but also disseminate information which include design requirements, design model, simulation videos, picture and so on. Consequently, production implementation would be completed better.

From time to time, process conditions need to be updated frequently. As a consequence, in order to avoid the wrong operation, bidirectional communication between process design system and production implementation system should be built and achieve the objective that process change and production

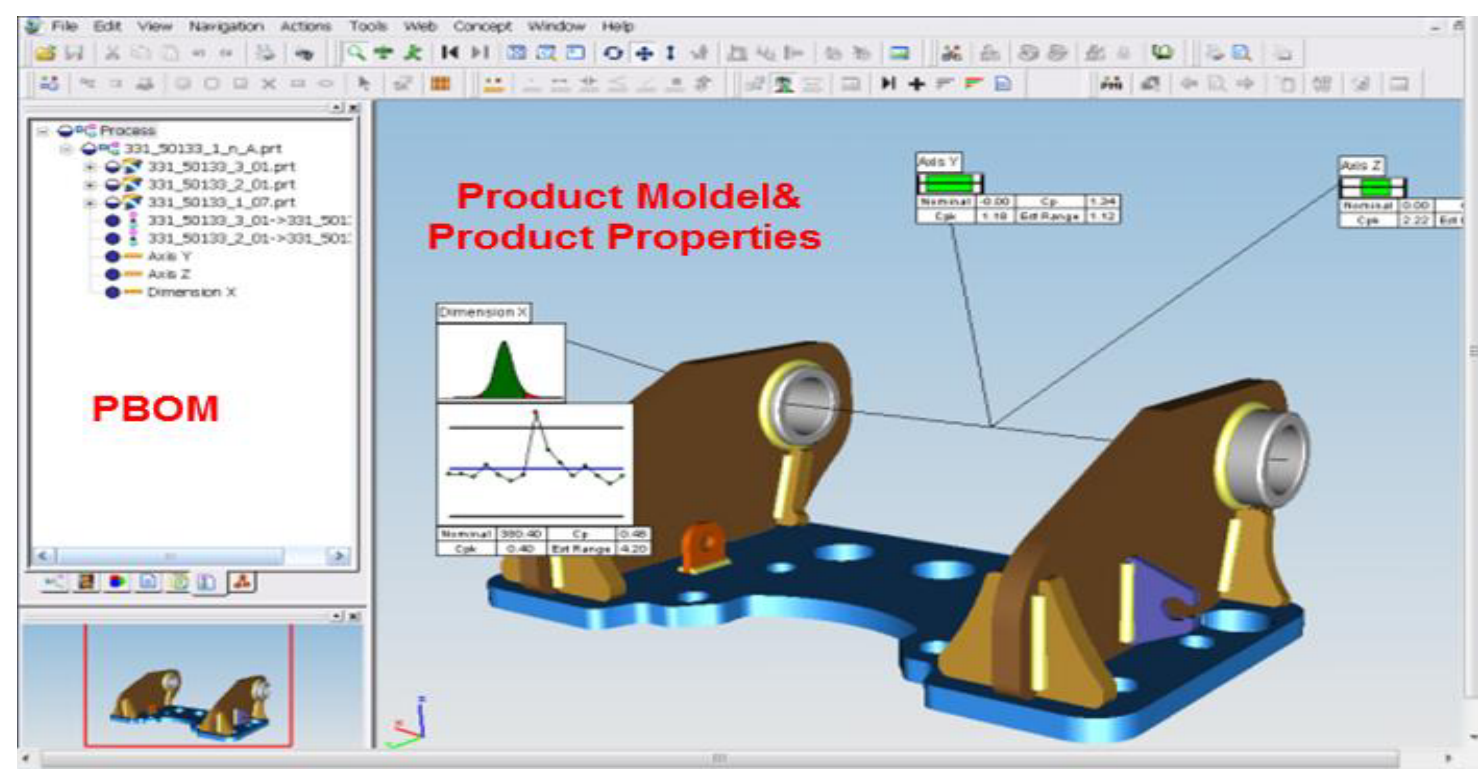

Fig.2. Assembly Process Digital Mock-up

structured process design. The core condition of bidirectional relationship is $3 \mathrm{D}$ structured process control of technology condition and collection of production parameters automatically.

\subsection{Bidirectional relationship between process design and production implementation}

implementation bidirectional lock and unlock synchronously.

Collection the production data automatically is key point for 3D spacecraft assembly. To achieve the goal nothing but building bidirectional relationship is feasible. Such a process is meant to prevent inaccurate data getting published to live production systems which may lead to wrong decisions. 


\subsection{Structured control technical conditions}

Traditional 3D spacecraft assembly was simple digitization by system which could produce digit data (such as CAPP, kanban system). Data package can't be produced automatically. Performs root cause analysis, non-structural control like text hinder progress for 3D spacecraft assembly. Using 3D spacecraft assembly mode, combination of design data, process data and production data offers an efficient means of controlling and building 3D spacecraft assembly data packets. Such combinations can be tuned and optimized to match 3D spacecraft assembly. Building structured parameters which explains the assembly production condition control production condition (for example, instrument $\mathrm{XX}$ is installed, grounding resistance value). Therefore, on the basis of analyses of various type structured parameters on process files, structure of technical conditional controls would be built. At the same time, relevance between technical conditional controls and 3D process files is committed to build relevance between 3D process files and production data. Sequentially, it is possible to collect production data automatically.

\section{Design of 3D structured process planning}

Design of 3D structured process planning as illustrated in the following figure. General research approach as following: Inheritance and reconstruction of process digital mock-up of prototype design is only data source. Firstly, design and simulation for process planning should be carried out. Secondly, technological process that had been verified by simulation would be designed and compiled minutely. Information about material like manufacturing resources, assembly relationship and engineering requirements can be obtained from process digital mock-up. Then, relevant process file, including product model, video, picture and so on, would be examined, approved and sent to MES system as foundation of production and decision-making.
According to[7],process digital mock-up not only include information about spacecraft, but also include implement, tools and process protecting parts which had been added by process itself. Meanwhile, combination with requirements of design, technological analysis and process planning can be accomplished.

First of all, using implement, tools, visual virtual human and visual virtual factory as data source, we can build all elements in the assembly simulation environment by 3D model of spacecraft. Simulation software use for design and verify technological process. Video simulation can be used for production and decision-making.

Then, Detailed process include grouping of process planning and the detailed design process route. It would be accomplished according to verified technological process or it may intersect with the verified technological process. Consequently, a better process planning was obtained.

\subsection{D assembly process compiling}

3D assembly process compile many aspects include proposing manufacturing resource requirements, process requirement description by text or reference process knowledge base, defining the implementation records, adding multimedia process information and accessories, processing method and so on. Working process is as follows:

- Reference process BOM, ensure Loading parts for current process are available.

- Reference process BOM or process knowledge library, ensure tools and auxiliary material for current process are available.

- Process attribute judgment, add text "critical process" to corresponding process.

- Reference process knowledge library(for example SOP, standard operation procedure),according to process planning and technical requirements compile assembly operation process text, and add marks about corresponding key inspection points and Compulsory

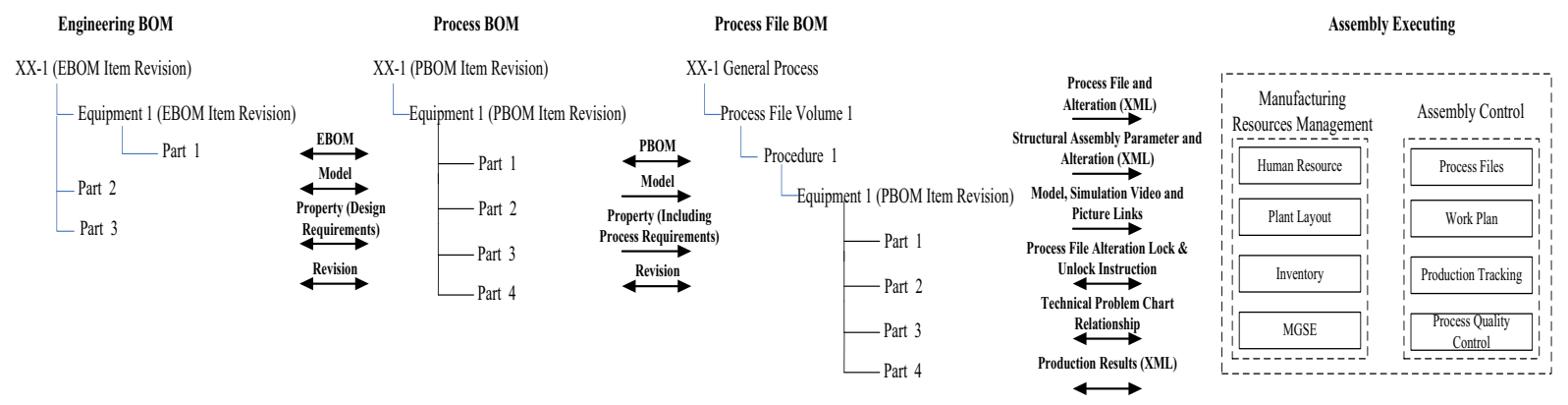

Fig.3. Scheme of 3D Structural Process Design

Finally, after instruction, material and staves had been signed and recorded by MES system, relevant manufacturing information would be sent back to process design system as assembly data packets.

\subsection{D assembly process planning}

inspection points.

- Defined a structured implementation records

- Adding multimedia(production model, video, picture and so on) process information to process accessories

- Automatic dispatch information to MES system after process documents had been signed and controlled. 


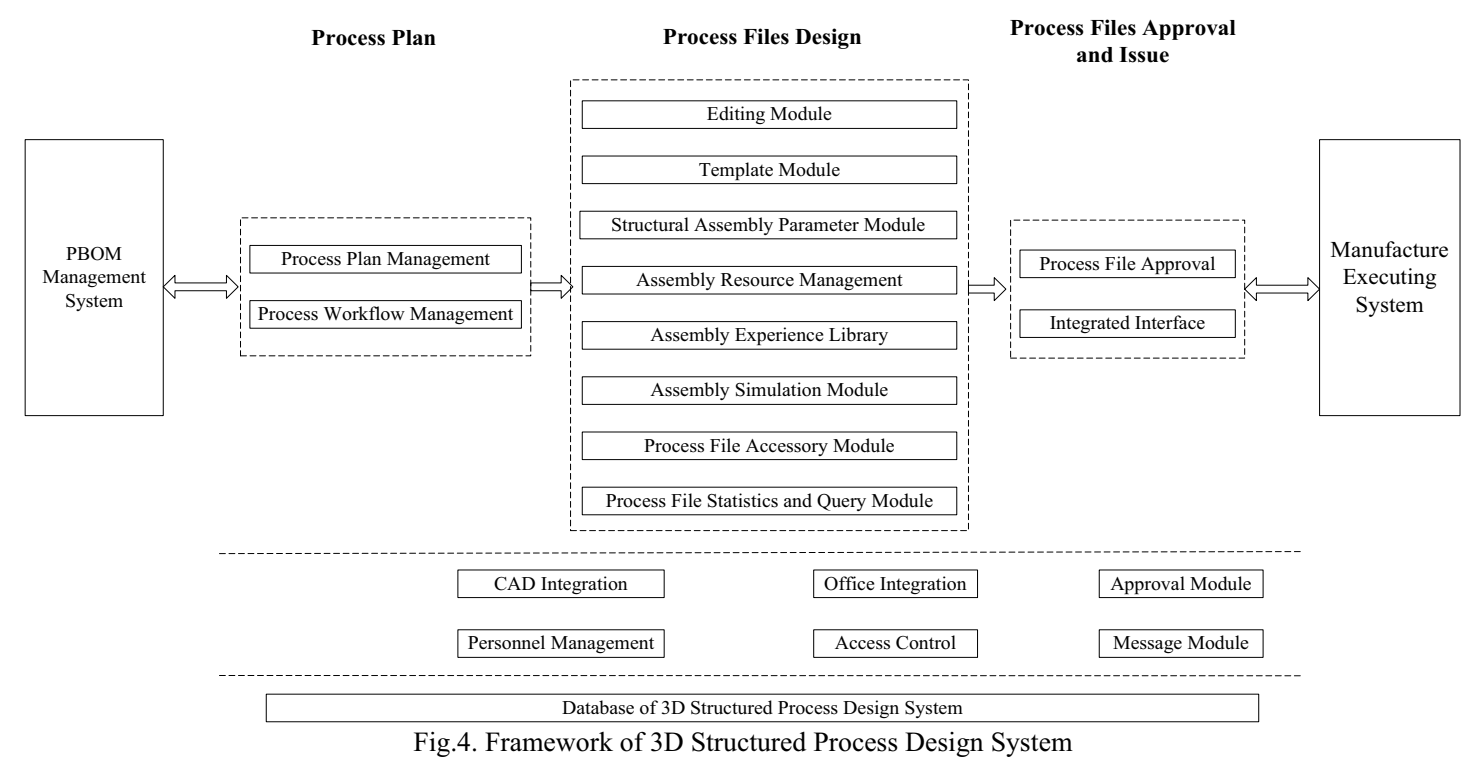

\subsection{Bidirectional integration with MES system}

3D process system send MES system information include the following: resources demanding information about materials, tools and so on; process requirement information such as PMI; multimedia(production model, video, picture and so on) process information.

In MES system, assembly production process for spacecraft as following:

- The starting point for distributed in 3D process, according to the process of form a complete set table warehouse operator start to collect and give out materials.

- According to the production plan, dispatcher distribute production task base on the module process and dynamic regulate of the control of the practical situation,

- After receiving the production task, production team start to assembly implementation and inspection based on the module process by means of checking the MES system of $3 \mathrm{D}$ process, and records the production condition and parameters before completion.

- After operation completed, inspector sign and record relevance process which include feedback implementation parameters of executive logging (for instance, the ground resistance) by means of checking the Real-time online MES system. Then system would sent process file with sign records and implementation parameters to $3 \mathrm{D}$ process system as whole satellite assembly data source by means of bidirectional integration interface.

- For sign records and implementation parameters produce on mobile termination, before 3D process system get relevant data, it would be collected by the real-time online MES system.

While the process is changing, 3D process system send lock instruction to the real-time online MES system and reminder users in relevant process. After the process change is completed, 3D process system send unlock instruction to the real-time online MES system and reminder users in relevant process and change the state of executable.

\section{Framework}

For system described above, you can count process digital mock-up system as data input interface of 3D structured process system mentioned in this paper. Data output interface can be regard as MES system including process planning module, process documentation, process of examination and approval and so on.

Process digital mock-up and its affiliated product are cited by association relationship between 3D structured process system and process digital mock-up system. However bidirectional communicate synchronously using website service between 3D structured process system and MES system by process, process update and executive logging.

According to technical solution as is mentioned above, a peculiar 3D structured process system for spacecraft was designed using Teamcenter from Siemens as a tool, and it was applied to several application about satellite development. In the meantime, we get 3D structured process based on uniform data source from assembly digital mock-up. Assembly process planning, assembly process documentation and distribution and coordinate closely with MES system had been researched and manufactured for spacecraft model XX. Consequently, completed assembled and production for spacecraft model XX, we generate data packets for whole satellite assembly.

\section{Conclusion}

In this paper we presented a concept of assembling of spacecraft. We focuses on designing a peculiar 3D assembly method meeting the demands of spacecraft. Current situation about digitization assembling for spacecraft development is explained. Furthermore, using uniform data source from assembly digital mock-up and analyzing two essential criteria about digitization assembling spacecraft, the solution was proposed for structured process design, working process and system framework about assembling spacecraft. Combined with 
the actual case demonstrates it has good application outcome.

\section{Acknowledgement}

This research is supported by Natural Science Foundation of China (51405025).

\section{References}

1. Lian Ding, Dannie Davies, Christopher A. McMahon. The integration of lightweight representation and annotation for collaborative design representation. Res Eng Design. 2009,20:185-200.

2. Zorriassatine F, Wykes C, Parkin R, et al. A survey of virtual prototyping techniques for mechanical product development $[\mathrm{J}]$. Proceedings of the Institution of Mechanical Engineers, Part B (Journal of Engingeering Manufacture). 2003, 217(B4):513530 .

3. Lihong Qiao, Jin Zhang. Some Key Issues in ThreeDimensional Digital Process Planning and Their Research. Aerospace Manufacturing Technology. 2010(1):29-32.

4. Tao Yang, Zhongyi Mei. Aircraft Full ThreeDimensional Implementation Technology Based on Mbd. Aerospace Manufacturing Technology. 2013(08):26-31.

5. Yuqing Fan, Yan Liang, Guocheng Feng. Data Organization of MBD-Based Assembly and Its Implementation, Aviation Maintenance \& Engineering .6(2010)56-59.

6. Sebastien Gagne, Clement Fortin. Application of the CMII Model to an integrated engineering and manufacturing development environment. Ingt $\mathrm{J}$ Ineract Des Manuf. 2007(1):5-13.

7. Wenxing He, Bile Wan, Bin Zhang, Xinghui Wu. Research of the technology and application of process digital mock-up for spacecraft assembly. Advanced Materials Research. 2014(1037):447-452.

8. In-Ho Song, Sung-Chong Chung. Synthesis of the digital mock-up system for heterogeneous CAD assembly. Computers in Industry. 2009(60):285-295.

9. Haiyan Wang. The MBD-based Final Assembly Process Planning Data Semantic Modeling Technology Research of Civil Aircraft [D]. Shanghai Jiao Tong University. 2013.

10. Yigguang Li, Zhiyi Pan, Ruijie Yan, Wenhe Liao. APDM-based framework for collaborative aircraft tooling design. International Journal of Production Research. 2008(46):2413-2431.

11. Yingguang Li, Zhiyi Pan, Ruijie Yan, Jianbang Jian. A semantics-based approach for collaborative aircraft tooling design. Advanced Engineering Informatics. 2010(24):149-158. 